\title{
Disposable contact lens use as a risk factor for microbial keratitis
}

\author{
Cherry F Radford, Darwin C Minassian, John K G Dart
}

\begin{abstract}
Aims-A case-control study was performed to evaluate soft contact lens (SCL) wear modality as a risk factor for microbial keratitis.

Methods-Contact lens wearers presenting as new patients to Moorfields Eye Hospital accident and emergency department during a 12 month period completed a self administered questionnaire detailing demographic data and contact lens use habits. Cases were patients with a clinical diagnosis of SCL related microbial keratitis. Controls were SCL users attending with disorders unrelated to contact lens wear. Odds ratios (estimates of relative risks) and $95 \%$ confidence limits (CL) were calculated through multivariable logistic regression analysis.

Results-There were 89 cases and 566 controls. A substantially increased risk with 1-4 weekly disposable SCL compared with non-disposable SCL was identified among both daily wear (DW) (odds ratio $=3.51,95 \%$ CL 1.60-7.66, $p=0.002)$ and extended wear (odds ratio $4.76,95 \% \mathrm{CL}$ 1.52-14.87, $p=0.007$ ) users after adjustment for demographic, lens use and hygiene variables. Other significant factors among DW users were "occasional" overnight use, use of chlorine based (as opposed to other chemical) systems in combination with poor storage case hygiene, and irregular disinfection.

Conclusion-Properties of some disposable SCL may be partly responsible for these excess risks. It is also possible, however, that this finding is largely a reflection of widespread complacency among patients and practitioners with respect to disposable SCL fitting and use.

(Br F Ophthalmol 1998;82:1272-1275)
\end{abstract}

Microbial keratitis used to be a complication associated with trauma or pre-existing ocular surface disease; contact lens wear, however, with its increasing popularity, has become the major predisposing factor. ${ }^{1}$

It is well established that overnight wear significantly increases the risk of corneal infection among soft contact lens (SCL) wearers. ${ }^{1-3}$ Disposable SCL were introduced to reduce the risk of SCL related complications, but casecontrol studies in the $\mathrm{UK}^{4}$ and $\mathrm{USA}^{5}$ subsequently showed an excess risk of microbial keratitis associated with these lenses. Neither study, however, was able to show a significant difference in risk between the two modalities used with the same wear schedule, and, although re-analysis ${ }^{3}$ of the USA study identified an increased risk (odds ratio 3.21, 95\% confidence limits (CL) 1.22-14.36) with disposable SCL after controlling for overnight use, the authors hypothesised that this was due to classification error with respect to overnight use among their subjects. A more recent study ${ }^{6}$ reports an excess risk (odds ratio 8.35, 95\% CL 1.08-64.46) of focal corneal infiltrates with overlying fluorescein staining among extended wear (EW) patients using disposable as opposed to reusable SCL. This was attributed, however, to a tendency towards more prolonged periods of continuous use among their EW patients using disposable lenses. Meanwhile, population based incidence studies conducted in Sweden ${ }^{7}$ found similar or reduced risks of microbial keratitis among daily wear (DW) users of disposable as opposed to non-disposable SCL. Their low incidence and severity of corneal infection, however, may limit the application of the Swedish results to other contact lens wearing populations.

This case-control study investigated the possibility of excess risk of microbial keratitis with disposable lenses themselves by calculating odds ratios (estimates of relative risks) through separate multivariable logistic regression analyses for DW and EW use.

\section{Methods}

DATA COLLECTION

Data were collected from contact lens wearers attending Moorfields Eye Hospital accident and emergency department as new patients during the 12 month period to 1 March 1993. Demographic, contact lens use, contact lens experience, and contact lens hygiene data were obtained by self administered questionnaire (the questionnaire is available on the BJO web site). Socioeconomic classification was carried out according to the 1991 census coding. ${ }^{9} \mathrm{EW}$ was defined as 24 hour contact lens use occurring at least once per week, although less frequent ("occasional") overnight wear was included in the analysis of DW patients. Disposable contact lenses were defined as contact lenses for disposal at intervals of between 1 and 4 weeks. Hygiene standards were evaluated as previously described, ${ }^{10}$ with each step in the hygiene regime being classified as optimal or suboptimal for the statistical analysis. Clinical data were taken from the hospital notes.

\section{CASES AND CONTROLS}

Cases were defined as patients with a clinical diagnosis of SCL related presumed microbial keratitis ${ }^{1}$; differential diagnosis by accident and 
Table 1 Characteristics of soft contact lenses (SCL) wearers studied for risk of microbial keratitis (MK). (Values are numbers (percentages) unless stated otherwise)

\begin{tabular}{|c|c|c|}
\hline & $M K$ cases $(n=89)$ & Controls $(n=566)$ \\
\hline Mean age (range) & $28.3(13-55)$ & $30.8(14-71)$ \\
\hline Ratio of males to females & $43(48)$ & $209(37)$ \\
\hline \multicolumn{3}{|l|}{ Social class based on occupation: } \\
\hline I-II & $51(57)$ & $345(61)$ \\
\hline III-V & $38(43)$ & $221(39)$ \\
\hline \multicolumn{3}{|l|}{ Experience of contact lens wear: } \\
\hline $0-5$ years & $57(64)$ & $315(56)$ \\
\hline$>5$ years & $32(36)$ & $251(44)$ \\
\hline \multicolumn{3}{|c|}{ Previous use of an alternative contact lens type: } \\
\hline No & $44(49)$ & $345(61)$ \\
\hline Yes & $45(51)$ & $221(39)$ \\
\hline \multicolumn{3}{|l|}{ Soft contact lens type: } \\
\hline Daily wear (DW) non-disposable & $34(38)$ & $426(75)$ \\
\hline DW disposable: & $23(26)$ & $86(15)$ \\
\hline Acuvue (Vistakon) & $20(23)$ & $58(10)$ \\
\hline Other & $3(3)$ & $28(5)$ \\
\hline Extended wear (EW) non-disposable: & $8(9)$ & $28(5)$ \\
\hline EW disposable: & $24(27)$ & $26(5)$ \\
\hline Acuvue (Vistakon) & $21(24)$ & $24(4)$ \\
\hline Other & $3(3)$ & $2(1)$ \\
\hline \multicolumn{3}{|c|}{ Unscheduled overnight use among DW patients: } \\
\hline No & $43(75)$ & $464(91)$ \\
\hline Yes $(<$ once per week $)$ & $14(25)$ & $48(9)$ \\
\hline \multicolumn{3}{|l|}{ Extent of overnight use among EW patients: } \\
\hline Mean nights in a row & 9.6 & 13.1 \\
\hline \multicolumn{3}{|l|}{ SCL disinfection: } \\
\hline Chlorine release systems & $24(27)$ & $114(20)$ \\
\hline Hydrogen peroxide & $27(30)$ & $283(50)$ \\
\hline Other chemical & $8(9)$ & $117(21)$ \\
\hline None & $15(17)$ & $37(6)$ \\
\hline Disposal on removal & $15(17)$ & $15(3)$ \\
\hline
\end{tabular}

Table 2 Relative risks of significant exposure factors for daily wear (DW) soft contact lens (SCL) related microbial keratitis, obtained through multivariable analysis

\begin{tabular}{lll}
\hline Exposure factor & Odds ratio $(95 \%$ CL) & p Value \\
\hline DW SCL wear modality: & 1.0 (referent) & \\
$\quad$ Non-disposable & $\mathbf{3 . 5 1}(1.60-7.66)$ & 0.002 \\
$\quad$ Disposable & 1.0 (referent) & \\
Unscheduled overnight use: & $\mathbf{3 . 9 5}$ (1.02-15.26) & 0.046 \\
$\quad$ No & 1.0 (referent) \\
Yes (< once per week) & $\mathbf{3 . 7 7 ( 1 . 4 2 - 9 . 9 8 )}$ & \\
Use of chlorine based disinfection in combination with & \\
$\quad$ Other chemical and poor case hygiene & 1.0 (referent) & \\
$\quad$ Chlorine and poor case hygiene & & \\
Disinfection frequency: & $\mathbf{2 . 0 6}(1.03-4.14)$ & 0.008 \\
$\quad$ Daily &
\end{tabular}

${ }^{\star}$ Owing to a significant interaction between disinfection type and case cleaning score, the data were stratified into "good" and "poor" case hygiene, and each stratum analysed separately.

Table 3 Multivariable analysis of daily wear (DW) use of Acuvue disposable lenses as a risk factor for microbial keratitis, in the main subset of patients (see text)

\begin{tabular}{lllll}
\hline Exposure factor & Cases & Controls & Odds ratio (95\% CL) & p Value \\
\hline DW SCL wear modality: & 26 & 384 & 1.0 (referent) & \\
Non-disposable & 14 & 42 & $4.07(1.74-9.54)$ & 0.001 \\
Acuvue & &
\end{tabular}

emergency ophthalmologists was assisted by clinical classification guidelines, incorporating signs and symptoms as well as size and location of lesions. ${ }^{11}$ Controls were SCL wearers attending with disorders unrelated to contact lens wear. Patients with a medical indication for contact lens wear were excluded from the study.

\section{STATISTICAL ANALYSIS}

Using software previously described, ${ }^{10}$ multiple logistic regressions were carried out to estimate the odds ratio(s) for each factor with optimal adjustment for possible confounding effects of all other variables included in the regression model, and to look for possible effect modification. The reported odds ratios are considered as estimates of relative risk and are interpreted
Table 4 Relative risks of lens wear modality for extended wear (EW) soft contact lens (SCL) related microbial keratitis, obtained through multivariable analysis

\begin{tabular}{lll}
\hline Exposure factor & $\begin{array}{l}\text { Odds ratio } \\
\text { (95\% confidence limits) }\end{array}$ & p Value \\
\hline $\begin{array}{l}\text { EW SCL wear modality: } \\
\begin{array}{l}\text { Non-disposable } \\
\text { Disposable }\end{array}\end{array}$ & $\begin{array}{l}1.0 \text { (referent) } \\
\mathbf{4 . 7 6}(1.52-14.87)\end{array}$ & 0.007 \\
\hline
\end{tabular}

as such in this paper. The strategy used in the multivariable regression modelling was similar to that described by Kleinbaum et $a l .{ }^{12}$ Separate models were used for DW and EW SCL users.

\section{Results}

CASES AND CONTROLS

Ninety three SCL users meeting study criteria presented with (presumed) microbial keratitis, but four were excluded owing to incomplete data. Forty four of 89 (49\%) cases were culture positive and/or classified as severe (central lesions more than $2 \mathrm{~mm}$ diameter). There were 566 control patients with complete data. Table 1 shows some characteristics of the study patients.

MULTIVARIABLE LOGISTIC REGRESSION ANALYSIS OF DAILY WEAR SOFT LENS USERS.

Compared with DW non-disposable contact lenses (the referent) DW disposable contact lenses were associated with a 3.51 times greater risk of microbial keratitis (Table 2). The main body of patients $(466 / 655,71 \%)$ were using DW non-disposable SCL or DW Acuvue disposable SCL, with no unscheduled overnight wear, and with SCL disinfectants limited to the three commonest types during the study period-hydrogen peroxide, thiomersal, or chlorine release. In this main subset, the relative risk for Acuvue disposable lenses was 4.07 (Table 3).

Other significant risk factors identified by multivariable analysis of the data for DW SCL users were "occasional" overnight use, use of chlorine based (as opposed to other chemical) systems in combination with poor storage case hygiene, and irregular disinfection (Table 2).

MULTIVARIABLE LOGISTIC REGRESSION ANALYSIS OF EXTENDED WEAR SOFT LENS USERS Compared with EW non-disposable contact lenses (the referent) EW disposable contact lenses were associated with a 4.76 times greater risk of microbial keratitis. Among EW patients no other factors showed evidence of a significant association (Table 4).

\section{Discussion}

A potential limitation of this study is the use of a possibly unrepresentative hospital population, although we feel that this is unlikely to have been an appreciable source of bias. ${ }^{1}$ One advantage of this population is that it provided a sample of patients from over 200 practices in a large catchment area, thereby allowing inference to a wide spectrum of the SCL wearing population. 
The study shows a substantially increased risk of microbial keratitis among both DW and EW users of disposable lenses, after adjustment for possible confounding factors. The findings contradict the results of some incidence studies. ${ }^{7}{ }^{813-15}$ These studies, however, either lacked sufficient statistical power to detect relative risks of the level found in this study $^{13-15}$ or were conducted in a country in which patient supervision and (perhaps consequently) the incidence and severity of corneal ulcers appear to differ markedly from that in other contact lens wearing populations. ${ }^{7-8}$ Two studies have already reported a similar or higher excess risk for the disposable modality, but have attributed it to underreported ${ }^{3}$ or more prolonged consecutive ${ }^{6}$ overnight use. Our multivariable analyses, which included adjustments for possible confounding effects of both unscheduled overnight wear among DW patients, and the number of days of continuous 24 hour wear among EW patients, suggests that the disposable modality itself is associated with an increased risk of microbial keratitis.

Acuvue (Vistakon) lenses were used by $72 \%$ (78/109) and 90\% (45/50) of DW and EW disposable SCL users respectively, and, among DW patients, we were able to show that the excess risk with disposables persists when these lenses are analysed separately. These lenses have a unique ionic, ultrathin, high water content design which may be related to their increased level of in vivo dehydration, ${ }^{16-18}$ a revolutionary manufacturing method that has been shown to be associated with an increased frequency of manufacturing defects, ${ }^{19}$ and rapid and distinctive tear protein deposition. ${ }^{20}$ The manufacturing technique has not been altered since the data were collected for this study. It is possible that some of these factors may increase the frequency of mechanical, immunological, and/or metabolic corneal compromise in wearers of this lens, thereby increasing the risk of corneal invasion by pathogenic organisms. Insufficient numbers prevented separate analysis of any of the other disposable lens types. It is therefore not possible to say how much of the excess risk associated with disposables is attributable to characteristics of Acuvue (and other brands) as opposed to the disposable modality itself. Patient and practitioner factors may be largely responsible for the excess risk. Potential factors include the use of disposable lenses as a panacea for poor hygiene compliance and/or depositing problems with other lens types, which may have increased the number of patients with these characteristics among the disposable users. The confounding effect of these factors, however, is likely to have been reduced by the inclusion of hygiene and lens wear history variables in the regression analysis. There are other factors, however, for which control was not possible. The intense marketing and commercial advantages of some disposables may have persuaded practitioners to fit patients for whom the available parameters were less than ideal, perhaps resulting in a lower standard of SCL fit among disposable lens wearers. Also, the emphasised convenience and promotion of increased safety may have tempted some patients to wear disposable lenses under adverse conditions, and may encourage attempts to self treat complications by lens replacement rather than seeking prompt professional advice.

It is hoped that the introduction and gaining popularity of daily disposable lenses will lower the incidence of microbial keratitis by removing the need for disinfection and storage case hygiene and reducing the attraction of overnight wear. Despite encouraging results among subjects who were carefully selected and monitored, ${ }^{212}$ small in number and followed for only 3 months, ${ }^{23}$ or reviewed retrospectively for ill defined corneal complications, ${ }^{24}$ the level of compliance with daily disposal among the general population is not yet known. If, as this study suggests, characteristics of some disposables and/or complacent attitudes to their fitting and use increase the risk of infection, convenience and a reduced incidence of minor complications ${ }^{21-24}$ may be the only advantages of daily disposables. Epidemiological studies are needed to assess the relative safety of this new modality with respect to corneal infection.

We thank the nursing and medical staff of Moorfields Eye Hospital accident and emergency department for their help in conducting the study, Mr M Matheson (microbiology, Institute of Ophthalmology), and Mr Ronald Martin for clerical assistance. The study was supported by grants from Bausch and Lomb, CIBA Vision, Vistakon, and Moorfields Eye Hospital. The authors have no financial interest in any of the products quoted.

1 Dart JKG, Stapleton F, Minassian D. Contact lenses and ther risk factors in microbial keratitis. Lancet 1991;338:650-3.

2 Schein OD, Glynn RJ, Poggio EC, et al. The relative risk of ulcerative keratitis among users of daily-wear and extended-wear soft contact lenses. N Engl F Med 1989;321: $773-8$.

3 Schein OD, Buehler PO, Stamler JF, et al. The impact of overnight wear on the risk of contact lens associated ulcerative keratitis. Arch Ophthalmol 1994;112:186-90.

4 Matthews TD, Frazer DG, Minassian DC, et al. Risks of keratitis and patterns of use with disposable contact lenses. Arch Ophthalmol 1992;110:1559-62.

5 Buehler PO, Schein OD, Stamler JF, et al. The increased risk Buehler PO, Schein OD, Stamler JF, et al. The increased risk
of ulcerative keratitis among disposable soft contact lens of ulcerative keratitis among disposable sot
users. Arch Ophthalmol 1992;110:1555-8.

6 Cutter GR, Chalmers RL, Roseman M. The clinical presentation, prevalence and risk factors of focal corneal infiltrates in soft contact lens wearers. CLAO F 1996;22:30-

7 Nilsson SEG, Montan PG. The hospitalised cases of contact lens induced keratitis in Sweden and their relation to lens type and wear schedule: results of a three-year retrospective study. $C L A O \mathcal{F} 1994 ; 20: 97-101$

8 Nilsson SEG, Montan PG. The annualised incidence of contact lens induced keratitis in Sweden and its relation to lens type and wear schedule: results of a 3-month prospective study. CLAO F 1994;20:225-30.

9 Office of Population Censuses and Surveys. Standard occupational classification. London: HMSO, 1990.
of

10 Radford CF, Bacon AS, Minassian DC, et al. Risk factors for Acanthamoeba keratitis in contact lens users: a casecontrol study. BMf 1995;310:1567-70.

11 Stapleton F, Dart JKG, Minassian DC. Risk factors with contact lens related suppurative keratitis. CLAO f 1993;19: 204-10.

12 Kleinbaum DG, Kupper LL, Morgenstern H. Modeling: analysis strategy. In: Epidemiologic research. London: Lifetime Learning Publications, 1982:447-56.

13 Poggio EC, Abelson MB. Complications and symptoms in disposable extended wear lenses compared with conventional soft daily wear and soft extended wear lenses. $C L A O$ f 1993;19:31-9.

14 Poggio EC, Abelson MB. Complications and symptoms with disposable daily wear contact lenses and conventional with daily wear contact lenses. CLAO f 1993;19:95-102.

15 Guillon M, Guillon JP, Bansal M, et al. Incidence of ulcers with conventional and disposable daily wear soft contact lenses. F Br Contact Lens Assoc 1994;17:69-76. 
16 Brennan NA, Lowe R, Efron N. In vivo dehydration of disposable (Acuvue) contact lenses. Optom Vis Sci 1990;67: posable

17 Helton DO, Watson LS. Hydrogel contact lens dehydration rates determined by thermogravimetric analysis. CLAO $\mathcal{F}$ 1991;17:59-61.

18 Pritchard N, Fonn D. Dehydration, lens movement and dryness ratings of hydrogel contact lenses. Ophthalmic Physiol Opt 1995;15:281-6.

19 Efron N, Veys J. Defects in disposable contact lenses can compromise ocular integrity. Int Contact Lens Clin 1992;19: 8-17.

20 Sack RA, Jones B, Antignani A, et al. Specificity and biological activity of the protein deposited on the hydrogel surface. Invest Ophthalmol Vis Sci 1987;28:842-9.
21 Nason RJ, Boshnick EL, Cannon WM. Multisite comparison of contact lens modalities. Daily disposable wear vs conventional daily wear in successful contact lens wearers. 7 Am Optom Assoc 1994;65:774-80.

22 Solomon OD, Freeman MI, Boshnick EL, et al. A 3-year prospective study of the clinical performance of daily disposable contact lenses compared with frequent replacement and conventional daily wear contact lenses. $C L A O \mathcal{F}$ $1996 ; 22: 250-7$

23 Nilsson SEG, Soderquist M. Clinical performance of a daily disposable contact lens: a three month prospective study. F Br Contact Lens Assoc 1995;18:81-6.

24 Hamano H, Watanabe K, Hamano T, et al. A study of the complications induced by conventional and disposable contact lenses. CLAO f 1994;20:103-8. 